\title{
Identification of key transcription factors in caerulein-induced pancreatitis through expression profiling data
}

\author{
DACHUAN QI ${ }^{1,2^{*}}$, BO WU ${ }^{2 *}$, DANIAN TONG ${ }^{2}$, YE PAN $^{2}$ and WEI CHEN ${ }^{2}$ \\ ${ }^{1}$ Department of Surgery, The First Affiliated Hospital of Soochow University, Suzhou, Jiangsu $215006 ;{ }^{2}$ Department of Surgery, \\ Shanghai Jiaotong University Affiliated Sixth People's Hospital, Shanghai 200233, P.R. China
}

Received February 14, 2014; Accepted November 7, 2014

DOI: $10.3892 / \mathrm{mmr} .2015 .3773$

\begin{abstract}
The current study aimed to isolate key transcription factors (TFs) in caerulein-induced pancreatitis, and to identify the difference between wild type and Mistl knockout (KO) mice, in order to elucidate the contribution of Mist1 to pancreatitis. The gene profile of GSE3644 was downloaded from the Gene Expression Omnibus database then analyzed using the t-test. The isolated differentially expressed genes (DEGs) were mapped into a transcriptional regulatory network derived from the Integrated Transcription Factor Platform database and in the network, the interaction pairs involving at least one DEG were screened. Fisher's exact test was used to analyze the functional enrichment of the target genes. A total of 1,555 and 3,057 DEGs were identified in the wild type and Mist1KO mice treated with caerulein, respectively. DEGs screened in Mist1KO mice were predominantly enriched in apoptosis, mitogen-activated protein kinase signaling and other cancer-associated pathways. A total of 188 and 51 TFs associated with pathopoiesis were isolated in Mist1 KO and wild type mice, respectively. Out of the top 10 TFs (ranked by P-value), 7 TFs, including S-phase kinase-associated protein 2 (Skp2); minichromosome maintenance complex component 3 (Mcm3); cell division cycle 6 (Cdc6); cyclin B1 (Ccnb1); mutS homolog 6 (Msh6); cyclin A2 (Ccna2); and cyclin B2 (Ccnb2), were expressed in the two types of mouse. These TFs were predominantly involved in phosphorylation, DNA replication, cell division and DNA mismatch repair. In addition, specific TFs, including minichromosome maintenance complex component 7 (Mcm7); lymphoid-specific helicase (Hells); and minichromosome maintenance complex component 6 (Mcm6), that function in the unwinding of DNA were
\end{abstract}

Correspondence to: Dr Dachuan Qi, Department of Surgery, The First Affiliated Hospital of Soochow University, 188 Shizi Road, Suzhou, Jiangsu 215006, P.R. China

E-mail: dachuanq@yeah.net

*Contributed equally

Key words: caerulein, transcription factor, pancreatitis, differentially expressed genes identified to participate in Mist1KO pancreatitis. The DEGs, including Cdc6, Mcm6, Msh6 and Wdr1 are closely associated with the regulation of caerulein-induced pancreatitis. Furthermore, other identified TFs were also involved in this type of regulation.

\section{Introduction}

Pancreatitis is an inflammatory disease associated with autodigestion of the gland. It is widely accepted that the injury is initiated within pancreatic acinar cells subsequent to premature intracellular activation of the digestive enzymes (1), which results in hydrops, hyperemia, hemorrhage and necrosis in the pancreas (2). In addition, acute pancreatitis may be associated with liver dysfunction and increased capillary permeability.

Caerulein is a polypeptide present in the brain and gastrointestinal tract, which has been demonstrated experimentally to result in pancreatitis. Caerulein administered via an intraperitoneal injection has been reported to induce acute edematous pancreatitis in mice, leading to the release of actin in pancreatic acinar cells, disruption to the transport of digestive enzymes and increased cellular permeability (3). In addition, several genes involved in this process have been identified: Cystathionine- $\gamma$-lyase serves an important pro-inflammatory role via nuclear factor $\kappa \mathrm{B}$ activation in caerulein-induced pancreatitis (4); S-propargyl-cysteine protects caerulein-induced acute pancreatitis via its involvement in the slow release of endogenous hydrogen sulfide (5); and neutral endopeptidase (NEP) is anti-inflammatory in caerulein-induced acute pancreatitis, with acute inhibition of NEP contributing to increased substance P levels (6).

It has previously been demonstrated that the knockout of certain TFs may also result in inflammation. For example, Mist1 (an exocrine-specific TFs) is important for the successful differentiation and functioning of pancreatic acinar cells (7). Another study demonstrated that Mist1 knockout $(\mathrm{KO})$ mice exhibited an altered stress response and increased sensitivity to caerulein-induced pancreatitis (8). In Mist1KO mice, multiple stress-associated genes, including c-Fos and c-Jun (together forming the activator protein 1 transcription complex), early growth response 1 , immediate early response 1, 2 and 5 and numerous heat shock proteins failed to accumulate in pancreatic tissue, suggesting a deficit 
in the ability to activate the stress response (8). However, the regulatory mechanism of these genes remains unclear.

In the current study, microarray data from caerulein-induced wild type and Mist1KO mice were used to isolate the differentially expressed genes (DEGs). Key regulatory TFs were then screened from regulatory networks that were constructed by DEGs, combined with the TFs in the database. Comparisons between the sample groups allowed the identification of important TFs, which may aid in the elucidation of the regulatory mechanisms in Mist1KO mice and contribute to knowledge of the pathogenic mechanisms underlying pancreatitis.

\section{Materials and methods}

Microarray data. The gene profile GSE3644 was downloaded from the Gene Expression Omnibus (http://www. ncbi.nlm.nih.gov/geo/). This expression dataset was created by Kowalik et al (8), who treated pancreases from wild type and Mist1KO mice with caerulein or saline as a control and processed them for RNA analysis. Targets from three biological replicates in each sample were generated and the expression profiles were determined using GeneChip Mouse Genome 430 Array (Affymetrix, Inc., Santa Clara, CA, USA). The 12 samples were obtained from three pancreases from each group (wild type saline, wild type caerulein, Mist1KO saline and Mist1KO caerulein) were used and biological replicates were analyzed.

Identification of DEGs. The t-test was used to analyze the gene expression profile in wild type and Mist1KO mice using the following formula (9):

$$
t=\frac{\bar{x}_{1}-\bar{x}_{2}}{\sqrt{\frac{\sigma_{x_{1}}^{2}+\sigma_{x_{2}}^{2}-2 \gamma \sigma_{x_{1}} \sigma_{x_{2}}}{n-1}}}
$$

$\overline{\mathrm{X}}_{1}$ and $\overline{\mathrm{X}}_{2}$ represent the average expression values of the gene in induced (caerulein-treated) and uninduced (saline-treated) groups; $\sigma_{\mathrm{X}_{1}}^{2}$ and $\sigma_{\mathrm{X}_{2}}^{2}$ are the variance of expression values under two different conditions; and $\gamma$ represents the correlation coefficient for genes between the induced and uninduced groups. Genes with significantly different expression $(\mathrm{P}<0.05)$ were isolated and considered to be DEGs. The Bayesian method (10) was used to adjust the raw P-values into the false discovery rate (FDR).

Construction of transcriptional regulatory network. A total of 34,679 transcriptional interaction pairs were isolated from the Integrated Transcription Factor Platform database (11), including 1,340 TFs and 4,785 target genes. Isolated DEGs were mapped into the transcriptional regulatory network, in which the interaction pairs involved at least one DEG were selected.

Functional enrichment analysis. Gene ontology functional enrichment analysis was conducted, using Database for Annotation, Visualization and Integrated Discovery

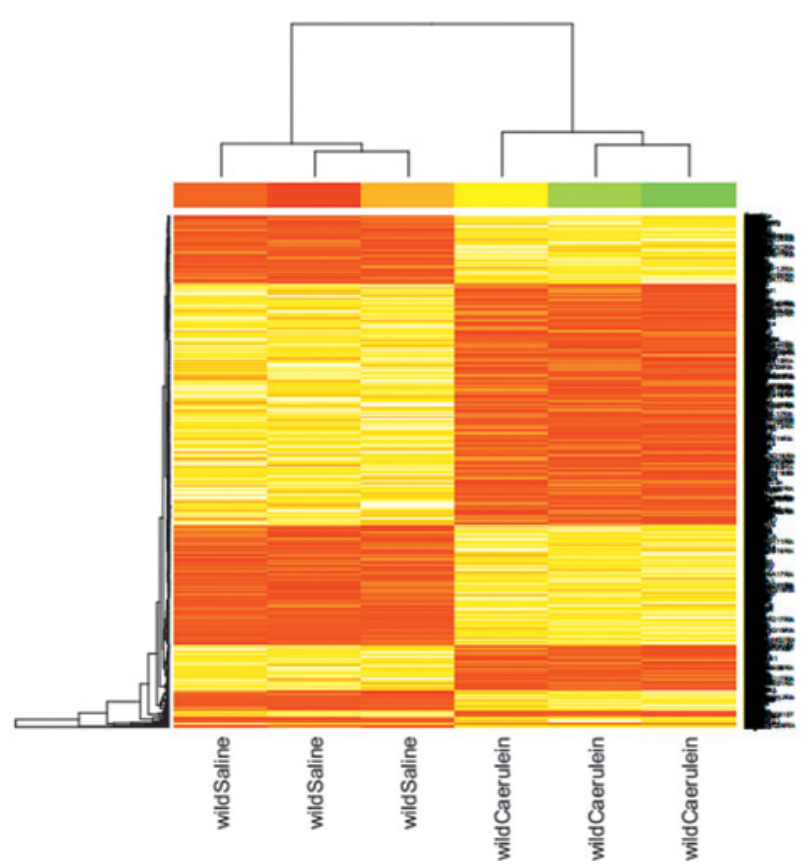

Figure 1. Two-way hierarchical clustering of differentially expressed genes in wild type mice. $\mathrm{y}$ axis, differentially expressed genes; $\mathrm{x}$ axis, samples.

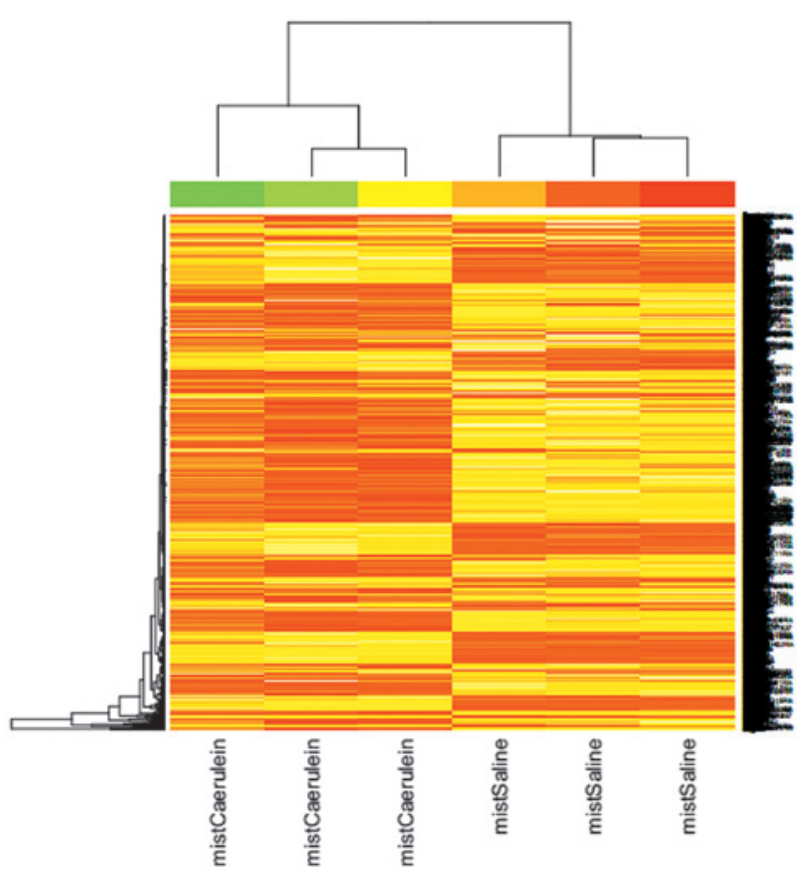

Figure 2. Two-way hierarchical clustering of differentially expressed genes in Mist1 knockout mice. y axis, differentially expressed genes; $x$ axis, samples.

(DAVID; http://david.abcc.ncifcrf.gov/) online software (12). Furthermore, two-way hierarchical clustering was conducted for the identified DEGs and the samples to distinguish the function of these DEGs, applying the gplots (13) package in R language (http://cran.r-project.org/web/packages/gplots/).

Enrichment analysis of gene sets. Fisher's exact test was used to analyze the enrichment of target genes using the following formula (14): 
Table I. Top 20 significantly enriched GO terms of the differentially expressed genes in wild type mice $($ FDR $<0.05)$.

\begin{tabular}{|c|c|c|}
\hline Category & Term & FDR \\
\hline GOTERM_CC_FAT & GO:0031090 organelle membrane & $6.40 \times 10^{-08}$ \\
\hline GOTERM_CC_FAT & GO:0005739 mitochondrion & $1.59 \times 10^{-06}$ \\
\hline GOTERM_CC_FAT & GO:0043233 organelle lumen & $3.84 \times 10^{-05}$ \\
\hline GOTERM_CC_FAT & GO:0031974 membrane-enclosed lumen & $5.99 \times 10^{-05}$ \\
\hline GOTERM_CC_FAT & GO:0070013 intracellular organelle lumen & $9.99 \times 10^{-05}$ \\
\hline GOTERM_CC_FAT & GO:0044429 mitochondrial part & $1.09 \times 10^{-04}$ \\
\hline GOTERM_CC_FAT & GO:0042470 melanosome & $9.80 \times 10^{-04}$ \\
\hline GOTERM_CC_FAT & GO:0048770 pigment granule & $9.80 \times 10^{-04}$ \\
\hline GOTERM_BP_FAT & GO:0022900 electron transport chain & 0.001402 \\
\hline GOTERM_CC_FAT & GO:0031975 envelope & 0.001806 \\
\hline GOTERM_MF_FAT & GO:0000166 nucleotide binding & 0.001984 \\
\hline GOTERM_BP_FAT & GO:0007242 intracellular signaling cascade & 0.002161 \\
\hline GOTERM_CC_FAT & GO:0031967 organelle envelope & 0.003051 \\
\hline GOTERM_CC_FAT & GO:0031988 membrane-bounded vesicle & 0.006726 \\
\hline GOTERM_CC_FAT & GO:0019866 organelle inner membrane & 0.006783 \\
\hline GOTERM_CC_FAT & GO:0012505 endomembrane system & 0.007954 \\
\hline GOTERM_CC_FAT & GO:0016023 cytoplasmic membrane-bounded vesicle & 0.008693 \\
\hline GOTERM_CC_FAT & GO:0031982 vesicle & 0.009787 \\
\hline GOTERM_CC_FAT & GO:0031410 cytoplasmic vesicle & 0.016995 \\
\hline GOTERM_CC_FAT & GO:0005743 mitochondrial inner membrane & 0.017543 \\
\hline
\end{tabular}

FDR, false discovery rate; GO, gene ontology.

Table II. Top 20 significantly enriched GO terms of the differentially expressed genes in Mist1 knock out mice (FDR<0.05).

\begin{tabular}{|c|c|c|}
\hline Category & Term & FDR \\
\hline GOTERM_CC_FAT & GO:0005739 mitochondrion & $9.65 \times 10^{-19}$ \\
\hline GOTERM_CC_FAT & GO:0044429 mitochondrial part & $6.55 \times 10^{-13}$ \\
\hline GOTERM_CC_FAT & GO:0031090 organelle membrane & $1.26 \times 10^{-11}$ \\
\hline GOTERM_CC_FAT & GO:0031967 organelle envelope & $2.94 \times 10^{-09}$ \\
\hline GOTERM_CC_FAT & GO:0031975 envelope & $3.85 \times 10^{-09}$ \\
\hline GOTERM_CC_FAT & GO:0048770 pigment granule & $3.06 \times 10^{-08}$ \\
\hline GOTERM_CC_FAT & GO:0042470 melanosome & $3.06 \times 10^{-08}$ \\
\hline GOTERM_CC_FAT & GO:0031974 membrane-enclosed lumen & $4.22 \times 10^{-08}$ \\
\hline GOTERM_BP_FAT & GO:0008104 protein localization & $6.11 \times 10^{-08}$ \\
\hline GOTERM_CC_FAT & GO:0043233 organelle lumen & $7.32 \times 10^{-08}$ \\
\hline GOTERM_CC_FAT & GO:0005783 endoplasmic reticulum & $7.53 \times 10^{-08}$ \\
\hline GOTERM_CC_FAT & GO:0070013 intracellular organelle lumen & $1.70 \times 10^{-07}$ \\
\hline GOTERM_BP_FAT & GO:0015031 protein transport & $3.98 \times 10^{-07}$ \\
\hline GOTERM_CC_FAT & GO:0005829 cytosol & $4.08 \times 10^{-07}$ \\
\hline GOTERM_CC_FAT & GO:0031980 mitochondrial lumen & $4.44 \times 10^{-07}$ \\
\hline GOTERM_CC_FAT & GO:0005759 mitochondrial matrix & $4.44 \times 10^{-07}$ \\
\hline GOTERM_BP_FAT & GO:0045184 establishment of protein localization & $6.95 \times 10^{-07}$ \\
\hline GOTERM_MF_FAT & GO:0000166 nucleotide binding & $6.38 \times 10^{-07}$ \\
\hline GOTERM_CC_FAT & GO:0012505 endomembrane system & $6.27 \times 10^{-07}$ \\
\hline GOTERM_CC_FAT & GO:0019866 organelle inner membrane & $4.02 \times 10^{-06}$ \\
\hline
\end{tabular}

FDR, false discovery rate; GO, gene ontology. 
Table III. Top 10 disease-associated transcription factors (ranked by P-value) in Mist1 knockout mice.

\begin{tabular}{|c|c|c|c|c|c|}
\hline Gene name & $\begin{array}{l}\text { DEGs } \\
\text { regulated }(n)\end{array}$ & $\begin{array}{l}\text { Non-DEGs } \\
\text { regulated (n) }\end{array}$ & $\begin{array}{c}\text { Total DEGs of all } \\
\text { transcription factors }(n)\end{array}$ & $\begin{array}{l}\text { Total non-DEGs of all } \\
\text { transcription factors }(n)\end{array}$ & P-value \\
\hline $\mathrm{Mcm} 7$ & 54 & 265 & 3,750 & 5,788 & $1.69 \times 10^{-17}$ \\
\hline Skp2 & 43 & 233 & 3,761 & 5,820 & $2.97 \times 10^{-17}$ \\
\hline $\mathrm{Mcm} 3$ & 25 & 176 & 3,779 & 5,877 & $1.25 \times 10^{-16}$ \\
\hline Cdc6 & 25 & 174 & 3,779 & 5,879 & $2.97 \times 10^{-16}$ \\
\hline Ccnb1 & 20 & 154 & 3,784 & 5,899 & $1.14 \times 10^{-15}$ \\
\hline Msh6 & 11 & 121 & 3,793 & 5,932 & $3.13 \times 10^{-15}$ \\
\hline Hells & 29 & 178 & 3,775 & 5,875 & $4.77 \times 10^{-15}$ \\
\hline Mcm6 & 34 & 193 & 3,770 & 5,860 & $4.79 \times 10^{-15}$ \\
\hline Ccna2 & 16 & 137 & 3,788 & 5,916 & $6.21 \times 10^{-15}$ \\
\hline Ccnb2 & 15 & 132 & 3,789 & 5,921 & $1.38 \times 10^{-14}$ \\
\hline
\end{tabular}

$\mathrm{P}<0.05$ was considered to indicate a statistically significant difference. DEGs, differentially expressed genes.

Table IV. Top 10 Disease-associated transcription factors (ranked by P-value) in wild type mice.

\begin{tabular}{|c|c|c|c|c|c|}
\hline Gene name & $\begin{array}{l}\text { DEGs } \\
\text { regulated } \\
\quad(n)\end{array}$ & $\begin{array}{l}\text { non-DEGs } \\
\text { regulated } \\
\text { (n) }\end{array}$ & $\begin{array}{l}\text { total DEGs of all } \\
\text { transcription factors } \\
\text { (n) }\end{array}$ & $\begin{array}{l}\text { total non-DEGs of all } \\
\text { transcription factors } \\
\text { (n) }\end{array}$ & P-value \\
\hline Mcm3 & 11 & 190 & 1,547 & 8,109 & $8.60 \times 10^{-06}$ \\
\hline Wdr1 & 46 & 107 & 1,512 & 8,192 & $8.92 \times 10^{-06}$ \\
\hline Skp2 & 20 & 256 & 1,538 & 8,043 & $2.44 \times 10^{-05}$ \\
\hline Ccna2 & 7 & 146 & 1,551 & 8,153 & $2.63 \times 10^{-05}$ \\
\hline Cdc6 & 12 & 187 & 1,546 & 8,112 & $3.18 \times 10^{-05}$ \\
\hline Cenf & 1 & 75 & 1,557 & 8,224 & $5.02 \times 10^{-05}$ \\
\hline Ccnb2 & 7 & 140 & 1,551 & 8,159 & $5.15 \times 10^{-05}$ \\
\hline Xbp1 & 15 & 18 & 1,543 & 8,281 & $5.47 \times 10^{-05}$ \\
\hline Ccnb1 & 10 & 164 & 1,548 & 8,135 & $8.47 \times 10^{-05}$ \\
\hline Msh6 & 6 & 126 & 1,552 & 8,173 & $9.38 \times 10^{-05}$ \\
\hline
\end{tabular}

$\mathrm{P}<0.05$ was considered to indicate a statistically significant difference. DEGs, differentially expressed genes.

$$
\mathrm{p}=\frac{\left(\begin{array}{c}
\mathrm{a}+\mathrm{b} \\
\mathrm{a}
\end{array}\right)\left(\begin{array}{c}
\mathrm{c}+\mathrm{d} \\
\mathrm{c}
\end{array}\right)}{\left(\begin{array}{c}
\mathrm{n} \\
\mathrm{a}+\mathrm{c}
\end{array}\right)}
$$

Where a represents a gene which is both a DEG and target gene of a TF; b represents a gene which is a target gene of a TF but not a DEG; c represents a gene which was a DEG but not a target of a TF; and d represents neither a DEG nor a target geen of a TF; $n$ represents the sum of $a, b, c$ and $d$.

\section{Results}

Identification of DEGs and enrichment analysis. A total of 9,856 genes were detected and 1,555 DEGs were identified between caerulein-induced and non-induced wild type mice, whilst 3,057 DEGs between caerulein-induced and non-induced Mist1KO mice were identified, using the t-test. In order to verify an association between these DEGs and pancreatitis, functional enrichment analyses were conducted. As demonstrated in Tables I and II, DEGs in wild type mice were predominantly interrelated with the functions of the cell membrane, signal transduction in the vesicle and cell membrane and molecular transport, all of which have a clear association with dysfunction of the pancreas acini. In addition to the wild type enriched functions, DEGs identified in Mist1KO mice were involved in apoptosis, mitogen-activated protein kinase (MAPK) signaling pathways and cancer-associated functions. In order to verify the function of these DEGs, two-way hierarchical clustering was conducted to compare the DEGs between different treatment groups in the original samples. Consequently, distinct differences between the induced and non-induced groups in the wild type and Mist1KO mice were observed (Figs. 1 and 2).

Construction and analysis of the transcriptional regulatory network. DEGs were mapped to the transcriptional regulation 


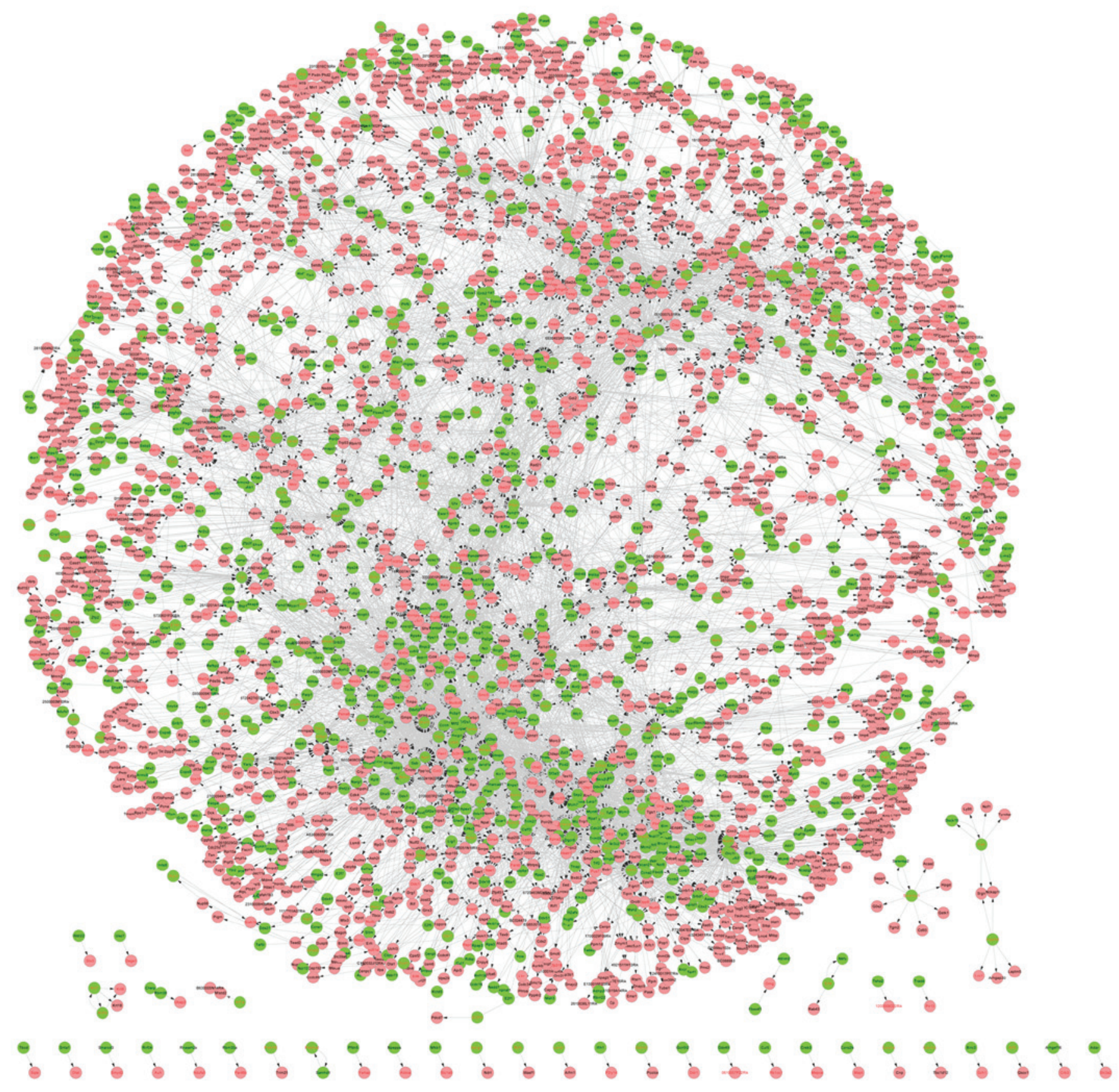

Figure 3. Transcriptional regulatory network of the differentially expressed genes in wild type mice. Green, transcription factors; pink, target genes.

database, then a transcriptional regulatory network was constructed. As demonstrated in Figs. 3 and 4, there were 8,913 interaction pairs in wild type mice and 14,684 interaction pairs of the transcriptional regulatory network in Mist1KO mice. The network in Mist1KO mice was larger than that of the wild type, indicating that there will be complex regulatory events Mist1KO mice subsequent to injection of caerulein. In target gene sets regulated by TFs, the enrichment of DEGs was calculated. The greater the DEG enrichment in the target gene was, the greater the likelihood that the TF was associated with the pathogenicity of caerulein. According to the statistical analysis, a total of 952 TFs were identified in the regulatory networks. Subsequent to screening using a P-value criterion of $0.01,188$ TFs were identified to be closely associated with pathopoiesis in Mist1KO mice, whereas $51 \mathrm{TFs}$ were identified in wild type mice. Functional enrichment with these TFs, indicated that the TFs in the Mist1KO mice were predominantly involved in the cell cycle and DNA replication and repair. In addition, TFs in wild type mice were involved in the cell cycle, cell adhesion and certain pathways associated with cancer. Among the top $10 \mathrm{TFs}$ in each group, 7 TFs were observed in both types of mice (wild type and Mist1KO), which included S-phase kinase-associated protein 2 (Skp2); minichromosome maintenance complex component 3 (Mcm3); cell division cycle 6 (Cdc6); cyclin B1 (Ccnb1); mutS homolog 6 (Msh6); cyclin A2 (Ccna2); and cyclin B2 (Ccnb2). In the Mist1KO mice, minichromosome maintenance complex component 7 (Mcm7), lymphoid specific helicase (Hells) and minichromosome maintenance complex component 6 (Mcm6) were observed, whereas in wild type mice, WD repeat domain 1 


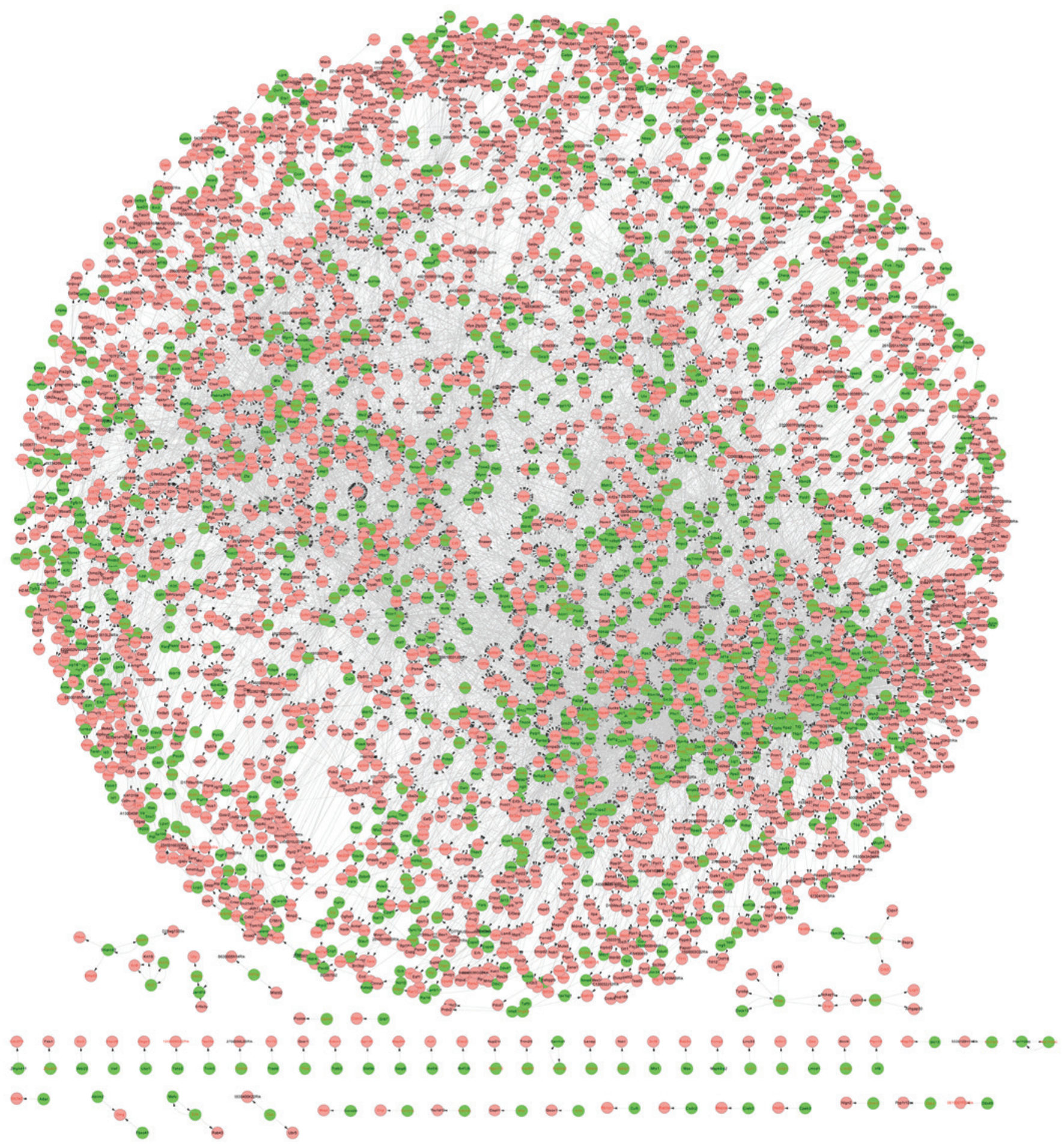

Figure 4. Transcriptional regulatory network in Mist1 knockout mice. Green, transcription factors; pink, target genes.

(Wdr1), cyclin F (Ccnf) and X-box binding protein 1 (Xbp1) were observed (Tables III and IV).

\section{Discussion}

Pancreatitis is a common pancreatic disease. Studies have demonstrated that caerulein may induce pancreatitis in mice and dysfunction in the Mistl gene may enhance inflammation and increase the sensitivity to caerulein-induced pancreatitis $(8,15,16)$. In the present study, a greater number of DEGs were identified between caerulein- and saline-treated Mist1KO mice compared with those observed in wild type mice. These DEGs were observed to be enriched in apoptosis, MAPK signaling and other cancer-associated pathways. Several key TFs were identified, a number of which were DEGs and may be able to regulate genes, such as Cdc6, Msh6, Wdr1 and Mcm6.

Cdc6 is an essential protein for the initiation of DNA replication (17), and may be highly prevalent in pancreatitis (18). It is reported that the induction of pancreatic acinar cell apoptosis is able to reduce the severity of pancreatitis $(19,20)$. Thus, high 
expression levels of Cdc6 may lead to increased pancreatic acinar cell proliferation and pancreatitis. Msh6 forms a heterodimer with MSH2 to produce a mismatch recognition complex that functions as a bidirectional molecular switch to exchange ADP and ATP, as DNA mismatches are bound and dissociated (21). Although the involvement of Msh6 in pancreatitis has not been clearly defined, it is considered that Msh6 is involved in the regulation of pancreatitis via DNA mismatch repair (22). In the current study, Cdc6 and Msh6 were identified as DEGs in wild type and Mist1KO mice, further indicating the importance of these two genes in caerulein-induced pancreatitis.

Wdr1 encodes a protein containing nine WD repeats (involved in protein-protein interactions) (23) and was identified in wild type mice. The encoded protein may aid in inducing the disassembly of actin filaments (24), which is considered to affect the secretion of pancreatic acinar cells $(25,26)$. Thus, Wdr1 is hypothesized to be involved in the regulation of pancreatitis through acinar cells.

In contrast, Mcm6 was observed in Mist1KO mice only. Mcm6, which belongs to the MCM family, is involved in the initiation of eukaryotic genome replication. The Mcm6 protein constructs an MCM complex with Mcm2, 4 and 7, which perform DNA helicase activity and may act to enzymatically unwind DNA (27,28). In addition, Mist1 is a basic helix-loop-helix TF and is involved in the maintenance of normal acinar cell function. A previous study reported that the expression of Mist1 results in a significant reduction in the proliferative potential of cells, whereas Mist1 knockdown leads to increased cell proliferation (7). Therefore, increased expression of Mcm6 may be due to the loss of Mist1 function, and thus enhances pancreatitis via increased proliferation.

In conclusion, high expression of Cdc6 and Mcm6 may lead to increased cell proliferation and pancreatitis. Msh6 and Wdr1 may be involved in the regulation of pancreatitis through DNA mismatch repair and acinar cells, respectively. Several key TFs that were not DEGs were also identified, these were considered to be involved in the regulation of caerulein-induced pancreatitis through target genes. Further investigation into these genes may contribute a more complete understanding of the regulatory network in pancreatitis and how this contributes to its pathogenic mechanisms.

\section{References}

1. Vonlaufen A, Wilson JS and Apte MV: Molecular mechanisms of pancreatitis: current opinion. J Gastroenterol Hepatol 23: 1339-1348, 2008.

2. Saluja AK and Steer ML: Pathophysiology of pancreatitis. Role of cytokines and other mediators of inflammation. Digestion 60 (Suppl 1): 27-33, 1999.

3. Ding SP, Li JC and Jin C: A mouse model of severe acute pancreatitis induced with caerulein and lipopolysaccharide. World J Gastroenterol 9: 584-589, 2003.

4. Ang AD, Rivers-Auty J, Hegde A, Ishii I and Bhatia M: The effect of CSE gene deletion in caerulein-induced acute pancreatitis in the mouse. Am J Physiol Gastrointest Liver Physiol 305 G712-G721, 2013.

5. Sidhapuriwala JN, Hegde A, Ang AD, Zhu YZ and Bhatia M: Effects of S-propargyl-cysteine (SPRC) in caerulein-induced acute pancreatitis in mice. PLoS One 7: e32574, 2012.

6. Koh YH, Moochhala S and Bhatia M: The role of neutral endopeptidase in caerulein-induced acute pancreatitis. J Immunol 187: 5429-5439, 2011.
7. Jia D, Sun Y and Konieczny SF: Mist1 regulates pancreatic acinar cell proliferation through p21 CIP1/WAF1. Gastroenterology 135: 1687-1697, 2008.

8. Kowalik AS, Johnson CL, Chadi SA, Weston JY, Fazio EN and Pin CL: Mice lacking the transcription factor Mist1 exhibit an altered stress response and increased sensitivity to caerulein-induced pancreatitis. Am J Physiol Gastrointest Liver Physiol 292: G1123-G1132, 2007.

9. Zhu M, Deng X, Joshi T, Xu D, Stacey G and Cheng J: Reconstructing differentially co-expressed gene modules and regulatory networks of soybean cells. BMC Genomics 13: 437, 2012.

10. Benjamini Y and Hochberg Y: Controlling the false discovery rate: a practical and powerful approach to multiple testing. J R Stat Soc Series B Stat Methodol 57: 289-300, 1995.

11. Zheng G, Tu K, Yang Q, et al: ITFP: an integrated platform of mammalian transcription factors. Bioinformatics 24: 2416-2417, 2008.

12. Huang DW, Sherman BT, Tan Q, et al: The DAVID Gene Functional Classification Tool: A novel biological module-centric algorithm to functionally analyze large gene lists. Genome Biol 8: R183, 2007.

13. Warnes GR, Bolker B, Bonebakker L, et al: gplots: Various R programming tools for plotting data. $\mathrm{R}$ package version $2: 4$, 2009.

14. Cantu E, Lederer DJ, Meyer K, et al; CTOT Investigators : Gene set enrichment analysis identifies key innate immune pathways in primary graft dysfunction after lung transplantation. Am J Transplant 13: 1898-1904, 2013.

15. Nathan JD, Romac J, Peng RY, Peyton M, MacDonald RJ and Liddle RA: Transgenic expression of pancreatic secretory trypsin inhibitor-I ameliorates secretagogue-induced pancreatitis in mice. Gastroenterology 128: 717-727, 2005.

16. Direnzo D, Hess DA, Damsz B, et al: Induced Mist1 expression promotes remodeling of mouse pancreatic acinar cells. Gastroenterology 143: 469-480, 2012.

17. Yan Z, DeGregori J, Shohet R, et al: Cdc6 is regulated by E2F and is essential for DNA replication in mammalian cells. Proc Natl Acad Sci USA 95: 3603-3608, 1998.

18. Salabat MR, Melstrom LG, Strouch MJ, et al: Geminin is overexpressed in human pancreatic cancer and downregulated by the bioflavanoid apigenin in pancreatic cancer cell lines. Mol Carcinog 47: 835-844, 2008.

19. Bhatia M, Wallig MA, Hofbauer B, et al: Induction of apoptosis in pancreatic acinar cells reduces the severity of acute pancreatitis. Biochem Biophys Res Commun 246: 476-483, 1998.

20. Bhatia M: Apoptosis versus necrosis in acute pancreatitis. Am J Physiol Gastrointest Liver Physiol 286: G189-G196, 2004.

21. Edelmann W, Yang K, Umar A, et al: Mutation in the mismatch repair gene Msh6 causes cancer susceptibility. Cell 91: 467-477, 1997.

22. Brentnall TA, Chen R, Lee JG, et al: Microsatellite instability and K-ras mutations associated with pancreatic adenocarcinoma and pancreatitis. Cancer Res 55: 4264-4267, 1995.

23. Mentel M, Iancu I, Szabo CZK, Munteanu CVA and Szedlacsek $\mathrm{S}$ : Co-expression of human Wdr1 gene with a chaperone increases its protein solubility. Rom J Biochem 50: 39-51, 2013.

24. Fujibuchi T, Abe Y, Takeuchi T, et al: AIP1/WDR1 supports mitotic cell rounding. Biochem Biophys Res Commun 327: 268-275, 2005.

25. Rosado JA, González A, Salido GM and Pariente JA: Effects of reactive oxygen species on actin filament polymerisation and amylase secretion in mouse pancreatic acinar cells. Cell Signal 14: 547-556, 2002.

26. Muallem S, Kwiatkowska K, Xu X and Yin HL: Actin filament disassembly is a sufficient final trigger for exocytosis in nonexcitable cells. J Cell Biol 128: 589-598, 1995.

27. Lee JK and Hurwitz J: Processive DNA helicase activity of the minichromosome maintenance proteins 4,6 , and 7 complex requires forked DNA structures. Proc Natl Acad Sci USA 98: 54-59, 2001.

28. Ohtani K, Iwanaga R, Nakamura M, et al: Cell growth-regulated expression of mammalian MCM5 and MCM6 genes mediated by the transcription factor E2F. Oncogene 18: 2299-2309, 1999. 